\title{
ALGUMAS TENDÊNCIAS DAS PUBLICAÇÕES RELACIONADAS À ASTRONOMIA EM PERIÓDICOS BRASILEIROS DE ENSINO DE FÍSICA NAS ÚLTIMAS DÉCADAS
}

Gustavo lachel*

Roberto Nardi**

RESUMO: Apresentamos neste artigo uma análise sobre as publicações relacionadas à Astronomia nas últimas décadas, presentes em dois periódicos de ensino de Física no país: o Caderno Brasileiro de Ensino de Física e a Revista Brasileira de Ensino de Física. Pelos conteúdos dessas publicações foi possível inferir algumas tendências da área de ensino de Astronomia, tais como: a frequência de publicações de artigos dessa natureza nesses periódicos no período analisado, a formação acadêmica dos autores que os publicaram, as temáticas relacionadas à Astronomia que aparecem nos artigos e as respectivas abordagens. Relacionamos alguns fatos ao crescimento da pesquisa no ensino da Astronomia no país.

Palavras-chaves: Ensino de Astronomia; Ensino de Física; Análise de Conteúdo; Estado da Arte.

\section{SOME TRENDS OF PUBLICATIONS RELATED TO ASTRONOMY IN PHYSICS EDUCATION JOURNALS IN THE LAST DECADES}

ABSTRACT: This paper was aimed at the analysis of the publications related to Astronomy in the last decades, present in two Physics Education journals in Brazil: Caderno Brasileiro de Ensino de Física and Revista Brasileira de Ensino de Física. Through the analysis of their contents it was possible to realize some trends in the Astronomy Education, such as: the frequency of papers of this area in these journals; the academic background of their authors, the subjects related to Astronomy and the approaches which appear in the papers analyzed. We connected some of these facts to the growth of the Astronomy Education research in the country.

Keywords: Astronomy Education; Physics Education; Content Analysis; Research; State of Art. 


\section{Introdução}

É comum pesquisadores de distintas áreas analisar as produções de seus pares em periódicos, anais de eventos, livros e demais veículos nos quais essas produções são disponibilizadas, com a finalidade de verificar as tendências da produção ou o aparecimento de novas linhas ou temáticas de pesquisa. Dependendo da profundidade e amplitude dessas revisões, bem como a utilização de referenciais teóricos pertinentes, esses estudos podem ser chamados de "estado da arte" de uma determinada área acadêmica.

André et al (1999), por exemplo, realizaram amplo estudo sobre as publicações relacionadas à formação de professores no Brasil. Nesse estudo, as autoras analisaram vários documentos como dissertações e teses (entre $1990 \mathrm{e}$ 1996), artigos de periódicos (entre 1990 e 1997) e trabalhos apresentados em eventos como a Anped" (entre 1992 e 1998), em uma tentativa de realizar "uma síntese integrativa do conhecimento sobre o tema da formação do professor" (ANDRÉ et al, 1999, p. 301).

Ostermann e Moreira (2000), por sua vez, investigaram as tendências da pesquisa sobre o ensino da Física Moderna Contemporânea, por meio de uma revisão da literatura que englobou os primeiros trabalhos publicados na área na década de 70, além de livros didáticos, dissertações, teses e textos na internet. Com isso, os pesquisadores puderam observar que neste campo de pesquisa,

Há uma grande concentração de publicações que apresentam temas de FMC [Física Moderna Contemporânea], em forma de divulgação, ou como bibliografia de consulta para professores do ensino médio. Por outro lado, existe uma escassez de trabalhos sobre concepções alternativas de estudantes acerca de tópicos de FMC, bem como pesquisas que relatam propostas testadas em sala de aula com apresentação de resultados de aprendizagem (OSTERMANN \& MOREIRA, 2000, p. 23).

Em outro estudo, Vasconcelos et al (2007) realizaram o "estado da arte" sobre a resolução de problemas em educação em ciências, analisando 69 artigos nacionais e internacionais (entre 2000 e 2003), com o objetivo de "recolher informação capaz de possibilitar a elaboração de um enquadramento teórico integrador do ensino por resolução de problemas" (VASCONCELOS, 2007, p. 235).

No caso específico do ensino de Astronomia, podemos citar trabalhos semelhantes como a revisão realizada por Bretones e Megid Neto (2003), sobre as tendências da área de Ensino de Astronomia a partir da análise de três teses e treze dissertações publicadas no Brasil, entre 1973 e 2002. Langhi (2004) também realizou extenso levantamento sobre as concepções alternativas de estudantes e professores sobre os diversos conteúdos relacionados à Astronomia. Mais recentemente, Marrone Júnior (2007), também produziu outra extensa revisão de artigos nacionais que tratam do ensino da Astronomia.

Visando colaborar para a continuidade e ampliação desses estudos, bem como contribuir com a memória da área de Educação em Astronomia, este 
artigo procura mostrar resultados de uma investigação sobre as tendências da pesquisa nesta área, a partir da análise dos artigos sobre Astronomia publicados nas duas últimas décadas (1990-2008) em dois periódicos nacionais da área de ensino de Física.

Para tanto, foram elaboradas algumas questões norteadoras que embasaram a análise dos artigos recolhidos:

a. Qual a frequência das publicações relacionadas à Astronomia nesses periódicos?

b. O que é possível afirmar sobre o perfil acadêmico dos pesquisadores que as publicaram?

balhos?

c. Quais são as abordagens utilizadas pelos pesquisadores em seus tra-

d. Que objetos de estudo ou conteúdos relacionados à Astronomia vêm sendo pesquisados?

\section{Detalhes sobre o estudo e a metodologia de recolha e análise dos dados}

Foram consultados dois periódicos nacionais para o levantamento dos artigos: o Caderno Brasileiro de Ensino de Física (CBEF) e a Revista Brasileira de Ensino de Física (RBEF). Esses periódicos foram selecionados por duas razões:

i. O tempo de existência desses periódicos, tornando-os consolidados e importantes no cenário acadêmico nacional;

ii. A inexistência de um periódico destinado ao Ensino de Astronomia que seja consolidado no cenário nacional. No momento, a RELEA (Revista Latino-Americana de Educação em Astronomia) completa apenas cinco anos e por essa razão não foi considerada no estudo descrito. Além disso, a Física e a Astronomia são ciências muito próximas. Alguns conceitos, como por exemplo, a gravitação universal, o campo magnético da Terra e a Astrofísica, comumente transitam entre essas duas ciências. De certa forma, isso justifica a quantidade de artigos relacionados à Astronomia publicados nesses periódicos.

Tabela 1: Algumas características dos periódicos consultados

\begin{tabular}{c|c|c|c|c|c} 
Periódico & $\begin{array}{c}\text { Site } \\
\text { Oficial }\end{array}$ & $\begin{array}{c}\text { Ano do } \\
\mathbf{1}^{\mathbf{0}} \text { Vol. }\end{array}$ & $\begin{array}{c}\text { Idade } \\
\text { (anos) }\end{array}$ & $\begin{array}{c}\text { Conceito no } \\
\text { WebQualis }\end{array}$ \\
\hline $\begin{array}{c}\text { Caderno Brasileiro de } \\
\text { Ensino de Física }\end{array}$ & $\begin{array}{c}\text { www.periodicos.ufsc.br/ } \\
\text { index.php/física }\end{array}$ & 1984 & 25 & $\begin{array}{c}\text { Universidade Federal de Santa } \\
\text { Catarina }\end{array}$ & B1 \\
\hline $\begin{array}{c}\text { Revista Brasileira de } \\
\text { Ensino de Física }\end{array}$ & www.sbfisica.org.br/rbef & 1979 & 30 & Sociedade Brasileira de Física & B1
\end{tabular}


Para a seleção dos artigos o critério utilizado foi apenas a presença de conteúdos relacionados à Astronomia nos referidos periódicos. Com isso, 58 artigos (26 do CBEF e 32 da RBEF, conforme o apêndice $A^{4}$ ) foram identificados, em consulta aos sites oficiais desses periódicos.

As publicações selecionadas foram submetidas à Análise de Conteúdo (BARDIN, 2000). A Análise de Conteúdo trata-se de um conjunto de técnicas que visam principalmente à ultrapassagem da incerteza, ou seja, investiga se a leitura que realizamos de uma mensagem é a mesma realizada pelas demais pessoas (se é generalizável); busca o enriquecimento da leitura, aumentando sua produtividade e pertinência.

A partir da Análise de Conteúdo de um texto, de uma conversa ou um documento, podemos recolher "indicadores" quantitativos ou qualitativos que nos possibilitem realizar "inferências de conhecimentos", ou "deduções lógicas" que, além de nos mostrar quais as condições de produção (de quem fala e de onde fala), podem também responder a questões de causa e efeito desses enunciados: "o que é que conduziu a um determinado enunciado?" e "quais as consequências que um determinado enunciado vai provavelmente provocar?" (BARDIN, 2000, p. 39).

O termo Análise de Conteúdo é definido pela autora, como um conjunto de técnicas que visa obter, por procedimentos sistemáticos e objetivos de descrição do conteúdo das mensagens, indicadores (quantitativos ou não) que permitam a inferência de conhecimentos relativos às condições de produção/ recepção (variáveis inferidas) dessas mensagens.

Como método de pesquisa, a Análise de Conteúdo é constituída de fases. A primeira, que diz respeito à organização da análise, "corresponde a um período de intuições, mas, tem por objetivo, tornar operacionais e sistematizar as ideias iniciais, de maneira a conduzir a um esquema preciso do desenvolvimento das operações sucessivas, num plano de análise" (BARDIN, 2000, p. 95). Nessa etapa, o pesquisador escolhe quais os documentos que pretende analisar. A exploração de todo o material ocorre na forma de leituras flutuantes, ou seja, aquelas nas quais o analista entra em contato com o objeto que será analisado e começa a formular algumas ideias iniciais. Durante a primeira fase também ocorre a elaboração de hipóteses, que são afirmações provisórias que desejamos verificar mediante análise, e os objetivos, que correspondem à finalidade da análise, ou seja, qual a questão que a análise do conteúdo pretende responder. Dessa forma, foram criadas hipóteses sobre as tendências da área, para serem investigadas. Com isso, é comum que ocorram as manifestações dos índices e indicadores (quantitativos e qualitativos).

Após a primeira fase da análise, quando ocorre a escolha dos documentos e a sua leitura flutuante, bem como a formulação de hipóteses, índices e indicadores, pode ocorrer a etapa de categorização dos componentes das mensagens analisadas. A autora afirma que "este processo não é uma etapa obrigatória de toda e qualquer Análise de Conteúdo", mas deixa claro que a "maioria dos 
procedimentos de análise organiza-se, no entanto, em redor de um processo de categorização" (BARDIN, 2000, p. 117). Com base nas questões que nortearam o estado da arte realizado, foram elaboradas categorias, cada qual com sua série de índices e indicadores, para melhor organizar as informações, contribuindo, dessa forma, para a realização das inferências.

Segundo Bardin, a categorização pode ser definida como

... uma operação de classificação de elementos constitutivos de um conjunto, por diferenciação e, seguidamente, por reagrupamento segundo o gênero (analogia), com os critérios previamente definidos. As categorias, são rubricas ou classes, as quais reúnem um grupo de elementos (unidades de registro, no caso da Análise de Conteúdo) sob um título genérico, agrupamento esse efetuado em razão dos caracteres comuns destes elementos (BARDIN, 2000, p. 117).

Como última etapa da Análise de Conteúdo, a interpretação das inferências consiste em conclusões pertinentes aos objetivos da pesquisa. O esquema abaixo apresentado foi elaborado para clarificar as etapas da Análise de Conteúdo realizada durante a pesquisa.

Figura 1: Esquema das etapas da Análise de Conteúdo realizada durante a pesquisa.

Adaptado de Bardin (2000, p. 102)

\section{Etapas da Análise de Conteúdo}

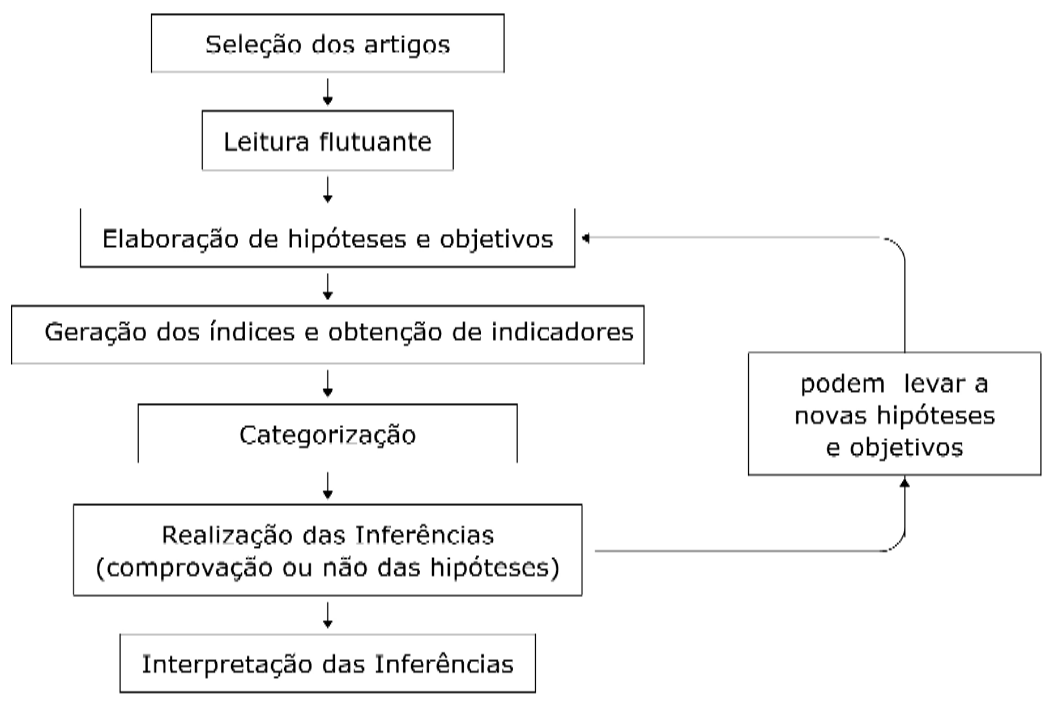

\section{Análise das publicações selecionadas}

Seguindo a metodologia descrita acima, a análise dos artigos foi dimensionada em quatro categorias, que descrevemos a seguir: 


\section{Categoria A: quantidade de publicações relacionadas à Astronomia}

Hipótese: o número de publicações relacionadas à Astronomia é maior a cada ano, o que indica que o número de pesquisadores envolvidos com essa Ciência vem aumentando no país.

Objetivo da análise: verificar a quantidade de publicações por ano, desde 1990 até 2008, como forma de buscar indicadores que apoiem (ou não) a hipótese.

Tabela 2: Número de publicações relacionadas à Astronomia, por ano

\begin{tabular}{c|c|c|c}
$\begin{array}{c}\text { Índice } \\
\text { Ano de publicaçäo }\end{array}$ & $\begin{array}{c}\text { Indicador } \\
\text { Quantidade de artigos selecionados }\end{array}$ & $\begin{array}{c}\text { Índice } \\
\text { Ano de publicação }\end{array}$ & Quantidade de artigos selecionados \\
\hline 1990 & 3 & 2000 & 7 \\
\hline 1991 & 0 & 2001 & 4 \\
\hline 1992 & 0 & 2002 & 3 \\
\hline 1993 & 2 & 2003 & 2 \\
\hline 1994 & 5 & 2004 & 2 \\
\hline 1995 & 0 & 2005 & 2 \\
\hline 1996 & 4 & 2006 & 4 \\
\hline 1997 & 4 & 2007 & 7 \\
\hline 1998 & 0 & 2008 & 5 \\
\hline 1999 & 4 & & $\mathbf{3 6}$
\end{tabular}

Figura 2: Gráfico da quantidade de publicações relacionadas à Astronomia, de 1990 até 2008

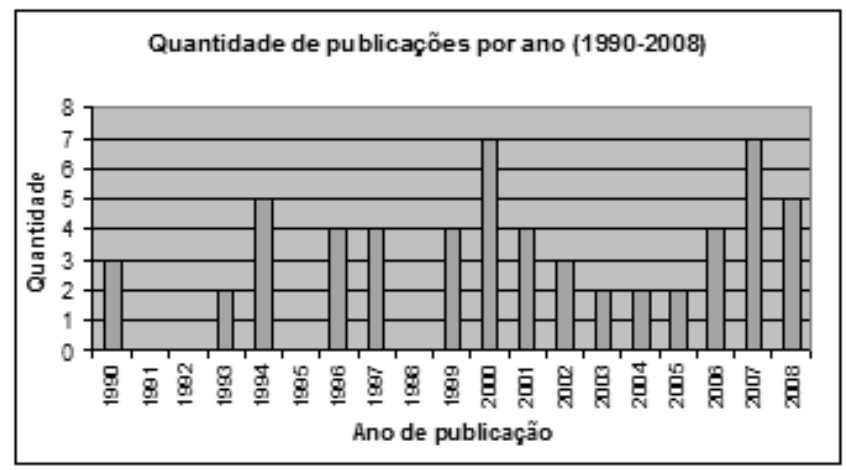

Inferências: nota-se claramente que o número de publicações foi maior na segunda década analisada. Além disso, a ocorrência de publicações tornou-se anual a partir de 1999, o que pode indicar consolidação da área de pesquisa relacionada à Astronomia no país. Além disso, em 1998 foram publicados os Parâmetros Curriculares Nacionais para o terceiro e quarto ciclos do ensino fundamental (BRASIL, 1998), que apresentaram uma série de conteúdos relacionados à Astronomia, o que pode ter incentivado a pesquisa desses temas e ocasionado o acréscimo das publicações. 


\section{Categoria B: perfil acadêmico dos pesquisadores (no ano da publicação)}

Hipótese: as primeiras publicações são de pesquisadores da área da Física, Astronomia e Astrofísica. Os pesquisadores das áreas de Educação e Ensino de Ciência passaram a contribuir com artigos relacionados à Astronomia a partir da gradativa consolidação da pesquisa em ensino nesta área.

Objetivo: além de investigar a formação dos autores, inferir sobre quando os pesquisadores na área de Educação e Ensino de Ciências começaram a colaborar com as pesquisas na área de Ensino de Astronomia.

Observação: foram identificados 84 autores. Suas formações acadêmicas foram consultadas no portal www.lattes.cnpq.br (foi considerado o ano de publicação). Dezesseis autores não possuíam cadastro no portal e não foram considerados nessa análise.

Tabela 3: Quantidade de autores por área

\begin{tabular}{c|c}
$\begin{array}{c}\text { Índice } \\
\text { Área }\end{array}$ & $\begin{array}{c}\text { Indicador } \\
\text { Quantidade de autores por área }\end{array}$ \\
\hline Astrofísica & 2 \\
\hline Astronomia & 12 \\
\hline Biociências & 2 \\
\hline Ciências & 3 \\
\hline Educação & 8 \\
\hline Ensino de Ciências & 4 \\
\hline Física & 24 \\
\hline Geofísica & 5 \\
\hline Matemática & 1 \\
\hline Ouímica & 2 \\
\hline Demais áreas & 5 \\
\hline Total & $\mathbf{6 8}$
\end{tabular}

Inferências: a análise aponta que os oito pesquisadores da área de Educação e os quatro da área de Ensino de Ciências publicaram seus artigos nos periódicos somente após o ano 2000 (artigos: CBEF2000b, CBEF2001b, CBEF2002a, CBEF2004a, CBEF2007b, CBEF2007c, RBEF2001b, RBEF 2006b, RBEF2007a e RBEF2008b, apresentados no apêndice A). Os autores da área de Astronomia, Astrofísica e Física colaboram desde os primeiros artigos analisados. Esse fato apoia a hipótese de que os autores da área de Educação e Ensino de Ciências colaboram com artigos relacionados à Astronomia, publicados nesses periódicos, há pouco tempo, a partir da gradativa consolidação da pesquisa em Educação em Astronomia.

\section{Categoria C: abordagens dos artigos}

Hipótese: a quantidade de artigos de abordagem teórica deve ser superior a quantidade de artigos relacionados ao Ensino de Astronomia, com abordagens no levantamento de concepções sobe Astronomia, análise de livros didáticos, formação de professores, tendo em vista que as áreas de Física, Astronomia e Astrofísica são mais antigas que as áreas de Educação e Ensino de Ciências. 
Objetivo: verificar a quantidade de artigos de acordo com sua abordagem.

Observação: foram identificadas as seguintes abordagens:

a. Desenvolvimento histórico do conteúdo relacionado à Astronomia: descrevem como o conhecimento relacionado à Astronomia se desenvolveu durante a história da humanidade;

b. Levantamento de concepções alternativas: esses estudos buscaram investigar o que os estudantes e/ou professores compreendem sobre alguns fenômenos astronômicos;

c. Experimental: são trabalhos que apresentam e avaliam experimentos e dinâmicas relacionados à Astronomia e a seu ensino;

d. Aprofundamento sobre conteúdos relacionados à Astronomia: é discutido, com maior profundidade, um determinado conceito relativo a essa Ciência. Muitos desses artigos apresentam equações relacionadas à Astronomia;

e. Análise de livros didáticos: apesar de os livros didáticos não incorrerem nos mesmos erros de uma década atrás, esses artigos são importantes, pois desmistificam muitos conceitos que se apresentavam de forma equivocada na época em que alguns professores atuantes ainda eram estudantes;

f. Formação de professores: apresentam atividades realizadas de formação inicial e continuada de professores.

Tabela 4: Quantidade de artigos identificados por sua abordagem

\begin{tabular}{c|c|c|c}
$\begin{array}{c}\text { Índice: } \\
\text { Abordagens }\end{array}$ & $\begin{array}{c}\text { Indicador: } \\
\text { Quantidade de artigos no CBEF }\end{array}$ & $\begin{array}{c}\text { Indicador: } \\
\text { Ouantidade de artigos na RBEF }\end{array}$ & $\begin{array}{c}\text { Indicador: } \\
\text { Quantidade total por abordagem }\end{array}$ \\
\hline $\begin{array}{c}\text { Análise de livro didático } \\
\text { Experimental }\end{array}$ & 4 & 0 & 4 \\
Formação de professores & 9 & 6 & 15 \\
Histórica & 1 & 1 & 2 \\
Levantamento de concepções & 5 & 2 & 7 \\
Teórica & 2 & 1 & 3 \\
Total & 11 & $\mathbf{2 6}$ & 27 \\
& $\mathbf{3 2}$ & $\mathbf{5 8}$
\end{tabular}

Inferências: o número de trabalhos teóricos é elevado em relação às demais abordagens, o que corrobora a hipótese elaborada. Além disso, nota-se que a RBEF possui muitos artigos dessa abordagem, e no CBEF, esse número aproxima-se da quantidade de artigos relacionados a experimentos relacionados à Astronomia.

\section{Categoria D: conteúdos relacionados à Astronomia abordados nos artigos}

Hipótese: Existe uma grande variedade de conteúdos relacionados à Astronomia que foram pesquisados durante essas duas últimas décadas. No entanto, deve haver temas que foram pouco explorados.

Objetivo da análise: investigar quais são os conteúdos pouco pesquisados, ou então, quais ainda não constaram nas contribuições a esses dois periódicos. 
Tabela 5: Conteúdos relacionados à Astronomia identificados nos artigos

\begin{tabular}{c|c}
$\begin{array}{c}\text { Índice } \\
\text { Conteúdo abordado }\end{array}$ & $\begin{array}{c}\text { Indicador } \\
\text { Número de artigos }\end{array}$ \\
\hline Sol & 4 \\
\hline Terra: constituição; forma; dimensão; rotação; precessão. & 7 \\
\hline Estações do ano & 1 \\
\hline Fases da Lua & 2 \\
\hline Eclipses solar ou lunar & 3 \\
\hline Equipamentos para observação celeste & 4 \\
\hline Estrelas, constelações elou aglomerados estelares & 5 \\
\hline Gnômon & 1 \\
\hline Gravitação & 2 \\
\hline Órbitas dos planetas / leis de Kepler & 5 \\
\hline Sistema Solar - sobre seus planetas ou sua formação & 2 \\
\hline Corpos menores do Sistema Solar (meteoroides, meteoritos, meteoros, cometas, asteroides) & 1 \\
\hline Cosmologia: formação do Universo; leis da Cosmologia; Astrofísica. & 9 \\
\hline Revolução copernicana & 3 \\
\hline Multidisciplinar & 9 \\
\hline Total & $\mathbf{5 8}$
\end{tabular}

Inferências: alguns conteúdos relacionados à Astronomia foram mais explorados por uma maior quantidade de pesquisadores, enquanto alguns tópicos como marés (abordado no artigo CBEF2003a), estações do ano (RBEF2007a), o uso do gnômon (RBEF1996a) e os corpos menores do Sistema Solar (CBEF1996a), são temas complexos e podem ser explorados em novas pesquisas. Além dos conteúdos identificados, é possível sugerir conteúdos relacionados à Astronomia que não foram identificados nesses periódicos: a criação dos calendários antigos, que foram baseados nos períodos solar ou lunar; a exploração espacial; noções do movimento aparente da esfera celeste; evolução da marcação do tempo, com a construção de relógios solares. Essas sugestões fazem parte dos PCNs para terceiro e quarto ciclos da educação básica (BRASIL, 1998).

\section{Algumas constatações}

A partir da análise de conteúdo dos artigos selecionados, é possível observar algumas tendências da área de Ensino de Astronomia:

- O número de publicações relacionadas à Astronomia cresceu nas últimas décadas, o que demonstra uma gradativa consolidação da área;

- O número de pesquisadores da área de Educação e Ensino de Ciências que se dedica a estudar essa temática também cresceu desde 2000;

- As abordagens que envolvem experimentação, além daquelas sobre formação de professores e levantamento de concepções alternativas relacionadas à Astronomia mostram, mais uma vez, o interesse da área de Educação e Ensino de Ciências pelo Ensino de Astronomia;

- Os conteúdos são diversificados, fato que consideramos importante para a consolidação da área de Ensino de Astronomia. 
Entendemos que o estudo descrito contribui para a gradativa consolidação da área de Educação em Astronomia, pois, além de ter objetivado a análise de tendências de artigos desse gênero em revistas nacionais, lista e divulga o acervo de documentos disponíveis aos professores e pesquisadores envolvidos com o ensino dessa ciência, conforme mostrado no Apêndice A.

\section{AGRADECIMENTOS}

Ao Prof. Dr. Rodolfo Langhi, pelas sugestões na elaboração deste estudo, e a CAPES e CNPq pelo apoio financeiro a este estudo e a outras pesquisas em desenvolvimento no Grupo de Pesquisa em Ensino de Ciências, da Unesp, Campus de Bauru. 


\section{Apêndice A}

\section{Quadro 1: Artigos selecionados no Caderno Brasileiro de Ensino de Física}

\begin{tabular}{|c|c|c|c|c|c|c|}
\hline Sigla & Autores & Título & Volume & Número & Página & Ano \\
\hline CBEF1990a & Livi, S.H.B. & Como medir a excentricidade da órbita da terra? (pense e responda) & 7 & 3 & 1.5 & 1990 \\
\hline CBEF1990b & Livi, S.H.B. & A Terra e o homem no universo & 7 & Esp & 7.26 & 1990 \\
\hline \multirow[t]{2}{*}{ CBEF1993a } & Perottoni, C.A., & Determinação da constante solar por meio de um calorímetro com gelo & 10 & 2 & $173 \cdot 178$ & 1993 \\
\hline & Zorzi, J.E. & & & & & \\
\hline CBEF1993b & Livi, S.H.B. & Eclipse solar total: 3 de Novembro de 1994 & 10 & 3 & $262 \cdot 268$ & 1993 \\
\hline CBEF1994a & Canalle, J.B.G. & 0 sistema solar numa representação teatral & 11 & 1 & 27.32 & 1994 \\
\hline CBEF1994b & Lopes, W. & O raio real do Sol & 11 & 2 & $115 \cdot 122$ & 1994 \\
\hline \multirow[t]{2}{*}{ CBEF1994c } & Garcia, J.B.G., & Comparação entre os tamanhos dos planetas e do Sol & 11 & 2 & $141 \cdot 144$ & 1994 \\
\hline & Oliveira, I.A.G. & & & & & \\
\hline CBEF1994d & Martins, R.A. & Galileo e a rotação da Terra & 11 & 3 & 196-211 & 1994 \\
\hline CBEF1994e & Canalle, J.B.G. & A luneta com lente de óculos & 11 & 3 & $212 \cdot 220$ & 1994 \\
\hline CBEF1996a & Damineli Neto, A. & Hale Bopp, o cometa do século? & 11 & 1 & $7 \cdot 10$ & 1996 \\
\hline CBEF1996b & Lamberti, P. W. & Astronáutica kepleriana & 13 & 2 & 154-164 & 1996 \\
\hline \multirow[t]{3}{*}{ CBEF1997a } & Trevisan, R.H., & Assessoria na avaliação do conteúdo de Astronomia dos livros de ciências do primeiro grau & 14 & 1 & $7 \cdot 16$ & 1997 \\
\hline & Lattari, C.J.B., & & & & & \\
\hline & Canalle, J.B.G. & & & & & \\
\hline CBEF1997b & Penereiro, J.C. & A filatelia como forma de divulgação da Astronomia & 14 & 1 & 64.82 & 1997 \\
\hline \multirow[t]{3}{*}{ CBEF1997c } & Canalle, J.B.G., & Análise do conteúdo de Astronomia de livros de Geografia do $1^{\circ}$ grau & 14 & 3 & $254-263$ & 1997 \\
\hline & Trevisan, R.H., & & & & & \\
\hline & Lattari, C.J.B. & & & & & \\
\hline CBEF1999a & Canalle, J.B.G. & Explicando Astronomia básica com uma bola de isopor & 16 & 3 & $314-331$ & 1999 \\
\hline \multirow[t]{2}{*}{ CBEF2000a } & Silveira, F.L., & Acerca de um mito: 0 vórtice de coriolis no ralo da pia & 17 & 1 & $22 \cdot 26$ & 2000 \\
\hline & Axt, R. & & & & & \\
\hline CBEF2000b & Neves, M.C.D. & A questão controversa da cosmologia moderna: Hubble e o infinito - parte 1 & 17 & 2 & 189-204 & 2000 \\
\hline CBEF2000c & Neves, M.C.D. & A questão controversa da cosmologia moderna: uma teoria e suas incongruências parte 2 & 17 & 2 & $205 \cdot 228$ & 2000 \\
\hline \multirow[t]{2}{*}{ CBEF2001d } & Saito, R.K., & Uma prática observacional em Astrofísica: o diagrama h-r de aglomerados abertos & 18 & 2 & $182 \cdot 196$ & 2001 \\
\hline & Baptista, R. & & & & & \\
\hline CBEF2001e & Harres, J. B. S. & A evolução do conhecimento profissional de professores: o caso do conhecimento prévio sobre a forma da Terra & 18 & 3 & $278-297$ & 2001 \\
\hline \multirow{2}{*}{ CBEF2002a } & Medeiros, A., & A invisibilidade dos pressupostos e das limitaç̃es da teoria copernicana nos livros didáticos de Física & 19 & 1 & 29.52 & 2002 \\
\hline & Monteiro, M. A. & & & & & \\
\hline CBEF2002b & Silveira, F. L. & A premissa metafísica da revolução copernicana & 19 & 3 & $407 \cdot 410$ & 2002 \\
\hline CBEF2003a & Silveira, F. L. & Marés, fases principais da Lua e bebês & 20 & 3 & $400-405$ & 2003 \\
\hline CBEF2004a & Hülsendger, M. & Uma análise das concepç̃os dos alunos sobre a queda dos corpos & 21 & 3 & $377 \cdot 391$ & 2004 \\
\hline \multirow[t]{2}{*}{ CBEF2005a } & Canalle, J.B.G., & Simplificando a luneta com lente de óculos & 22 & 1 & $121 \cdot 130$ & 2005 \\
\hline & Souza, A.C.F. & & & & & \\
\hline CBEF2005b & Andrade, N. L. & A revolução copernicana: considerações sobre duas questões do ENEM & 22 & 2 & $263-283$ & 2005 \\
\hline \multirow[t]{2}{*}{ CBEF2007a } & Dionisio, P.H., & Já lhe perguntaram... por que não existem estrelas verdes? & 24 & 1 & $50-53$ & 2007 \\
\hline & Dionísio, G. & & & & & \\
\hline \multirow[t]{3}{*}{ CBEF2007b } & Pinto, S. P., & Formação continuada de professores: estratégia para o ensino de Astronomia nas séries iniciais & 24 & 1 & 71.86 & 2007 \\
\hline & Fonseca, 0.M., & & & & & \\
\hline & Vianna, D.M. & & & & & \\
\hline \multirow[t]{2}{*}{ CBEF2007c } & Langhi, R., & Ensino de Astronomia: erros conceituais mais comuns presentes em livros didáticos de Ciências & 24 & 1 & $87-111$ & 2007 \\
\hline & Nardi, R. & & & & & \\
\hline \multirow[t]{3}{*}{ CBEF2007d } & Paula, L.A.N., & Uma contribuição à construção de espelhos parabólicos & 24 & 3 & $338-352$ & 2007 \\
\hline & Raggio, P., & & & & & \\
\hline & Assis, A.K.T. & & & & & \\
\hline \multirow[t]{2}{*}{ CBEF2007e } & Silveira, F.L., & 0 eclipse solar e as imagens do Sol observadas no chão ou numa parede & 24 & 3 & $353-359$ & 2007 \\
\hline & Axt, R. & & & & & \\
\hline \multirow[t]{2}{*}{ CBEF2008a } & Silveira, F.L., & 0 "encolhimento" das sombras & 25 & 2 & $228-246$ & 2008 \\
\hline & Saraiva, M.F.O. & & & & & \\
\hline
\end{tabular}


Quadro 2: Artigos selecionados na Revista Brasileira de Ensino de Física

\begin{tabular}{|c|c|c|c|c|c|c|}
\hline Sigla & Autores & Título & Volume & | Número & Página & Ano \\
\hline \multirow[t]{2}{*}{ RBEF1990a } & Fabris, J.C., & 0 momento quadrupolar do Sol e a precessão das órbitas elípticas & 12 & 1 & $26-45$ & 1990 \\
\hline & Silveira, F.E.M. & & & & & \\
\hline \multirow[t]{2}{*}{ RBEF1996a } & Evangelista, R. L., & Singularidades nuas e a precessão das órbitas elípticas & 18 & 1 & 7.13 & 1996 \\
\hline & Fabris, J.C. & & & & & \\
\hline RBEF1996b & Afonso, G.B. & Experiências simples com 0 Gnômon & 18 & 3 & $149 \cdot 154$ & 1996 \\
\hline \multirow{2}{*}{ RBEF1997a } & Miranda, P., & Simulação dos movimentos dos planetas - bacia de Kepler & 19 & 2 & 173 & 1997 \\
\hline & Weltner, K. & & & & & \\
\hline RBEF1999a & Kokubun, F. & A Lei de Hubble e a Homogeneidade do Universo & 21 & 3 & $311-313$ & 1999 \\
\hline \multirow{2}{*}{ RBEF1999b } & Oliveira, A.S., & Correção ao teorema de Stevin devido à rotação do planeta & 21 & 3 & 332.335 & 1999 \\
\hline & Amorim, A.E.A. & & & & & \\
\hline RBEF1999c & Tarsia, R.D. & 0 movimento de precessão da Terra & 21 & 4 & $507-511$ & 1999 \\
\hline RBEF2000a & Tavares, M. & Aprendendo sobre o Sol & 22 & 1 & 78.82 & 2000 \\
\hline RBEF2000b & Waga, I. & A expansão do Universo & 22 & 2 & $163 \cdot 175$ & 2000 \\
\hline RBEF2000c & Tavares, M. & Meteorologia Espacial & 22 & 4 & $496-502$ & 2000 \\
\hline RBEF2000d & Neves, M.C.D. & A Terra e a sua posição no Universo: formas, dimensões e modelos orbitais & 22 & 4 & $557-567$ & 2000 \\
\hline RBEF2001a & Ferreira, R. & Como medir 0 raio de curvatura da Terra com 0 auxílio de um canal de navegação & 23 & 2 & $236 \cdot 237$ & 2001 \\
\hline RBEF2001b & Silveira, F. L. & As variações dos intervalos de tempo entre as fases principais da Lua & 23 & 3 & $300-307$ & 2001 \\
\hline RBEF2002a & Fagundes, H.V. & Modelos cosmológicos e a aceleração do Universo & 24 & 2 & $247-253$ & 2002 \\
\hline \multirow[t]{7}{*}{ RBEF2003a } & Echer, E., & 0 número de manchas solares, índice da atividade do Sol & 25 & 2 & $157 \cdot 163$ & 2003 \\
\hline & Rigozo, N.R., & & & & & \\
\hline & Nodermann, & & & & & \\
\hline & D.J.R., & & & & & \\
\hline & Vieira, L.E.A., & & & & & \\
\hline & Prestes, A., & & & & & \\
\hline & Faria, H.H. & & & & & \\
\hline \multirow[t]{3}{*}{ RBEF2004a } & Dias, P.M.C., & A Gravitação Universal & 26 & 3 & 257.271 & 2004 \\
\hline & Santos, W.M.S., & & & & & \\
\hline & Souza, M.T.M. & & & & & \\
\hline \multirow[t]{3}{*}{ RBEF2006a } & Echer, E., & Ondas de choque não colisionais no espaço interplanetário & 28 & 1 & 51.66 & 2006 \\
\hline & Alves, M.V., & & & & & \\
\hline & Gonzales, W.D. & & & & & \\
\hline \multirow[t]{2}{*}{ RBEF2006b } & Scaracini, A.L., & Um curso de Astronomia e as pré-concepções dos alunos & 28 & 1 & 89.99 & 2006 \\
\hline & Pacca, J.L.A. & & & & & \\
\hline \multirow[t]{2}{*}{ RBEF2006c } & Dias, W.S., & A velocidade de rotação dos braços espirais da Via Láctea & 28 & 2 & $155 \cdot 160$ & 2006 \\
\hline & Lépine, J. & & & & & \\
\hline \multirow[t]{6}{*}{ RBEF2006d } & Bernardes, T.0., & Abordando o ensino de Óptica através da construção de telescópios & 28 & 3 & $391-396$ & 2006 \\
\hline & Barbosa, R.R., & & & & & \\
\hline & lachel, G., & & & & & \\
\hline & Batagin Neto, A., & & & & & \\
\hline & Pinheiro, M.A.L., & & & & & \\
\hline & Scalvi, R.M.F. & & & & & \\
\hline \multirow[t]{2}{*}{ RBEF2007a } & Dias, W.S., & Por que a variação da distância Terra-Sol não explica as estaçōes do ano? & 29 & 3 & 325.359 & 2007 \\
\hline & Piassi, L.P. & & & & & \\
\hline \multirow[t]{2}{*}{ RBEF2007b } & Filho, J.B.B., & A entropia de Hawking para buracos negros: um exercício de análise dímensional a partir de um texto de divulgação & 29 & 4 & 527.533 & 2007 \\
\hline & Araújo, R.M.X. & & & & & \\
\hline \multirow[t]{3}{*}{ RBEF2008a } & Silva, G.M.S., & Transformação de coordenadas aplicada à construção da maquete tridimensional de uma constelacăão & 30 & 1 & 1306 & 2008 \\
\hline & Ribas, F.B., & & & & & \\
\hline & Freitas, M.S.T. & & & & & \\
\hline \multirow[t]{4}{*}{ RBEF2008b } & Reis, N.T.0., & Análise da dinâmica de rotação de um satélite artificial: uma oficina pedagógica em educação espacial & 30 & 1 & 1401 & 2008 \\
\hline & Garcia, N.M.D., & & & & & \\
\hline & Souza, P.N., & & & & & \\
\hline & Baldessar, P.S. & & & & & \\
\hline \multirow[t]{4}{*}{ RBEF2008c } & Dominici, T.P., & Atividades de observação e identificação do céu adaptadas às pessoas com deficiência visual & 30 & 4 & 4501 & 2008 \\
\hline & Oliveira, E., & & & & & \\
\hline & Sarraf, $V_{\text {., }}$ & & & & & \\
\hline & Guerra, $F$. & & & & & \\
\hline \multirow[t]{3}{*}{ RBEF2008d } & Levenhagen, & A rotação estelar e seu efeito sobre os espectros & 30 & 4 & 4701 & 2008 \\
\hline & R.S., & & & & & \\
\hline & Künzel, R. & & & & & \\
\hline
\end{tabular}




\section{NOTAS}

1 Associação Nacional de Pós-Graduação e Pesquisa em Educação. Disponível em: $<$ http://www.anped.org.br> Acessado em Junho de 2009.

2 Conceito atribuído a esses periódicos, no chamado WebQualis, pela Área de Ensino de Ciências e Matemática na avaliação da Capes (Coordenação de Aperfeiçoamento de Pessoal de Nível Superior), que atribui conceitos aos periódicos, apenas para fins de avaliação da produção acadêmica de docentes e discentes credenciados em programas de pós-graduação da área; no caso, a área 46.

Fonte: <http://qualis.capes.gov.br/webqualis/Index.faces>, acessado em Junho de 2009.

3 Até o volume 18, número 3, o título do periódico era Caderno Catarinense de Ensino de Física.

4 Para cada trabalho foi designada uma sigla que se referencia ao periódico e ao ano de publicação (ex: CBEF2007c foi o terceiro artigo relacionado à Astronomia publicado em 2007 no CBEF).

5 Alguns trabalhos possuem vários temas abordados, como, por exemplo, aqueles que tratam do levantamento de concepções relacionadas à Astronomia, da formação de professores, da meteorologia espacial e de atividades práticas (ex: CBEF1999a).

\section{REFERÊNCIAS BIBLIOGRÁFICAS}

ANDRÉ, M.; SIMÕES, R. H. S.; CARVALHO, J. M. \& BRZEZINSKI, I., Estado da arte da formação de professores no Brasil, Educação \& Sociedade, ano XX, n. 68, Dez., 1999. Disponível em <http://www.scielo.br/pdf/es/v20n68/a15v2068.pdf >, acessado em Junho de 2009.

BARDIN, L. Análise de Conteúdo, Portugal: Edições 70, 225p., 2000.

BRASIL. Secretaria de Educação Média e Tecnologia. Parâmetros Curriculares Nacionais: terceiro e quarto ciclos do ensino fundamental - Ciências Naturais. Brasilia. MEC/SEMTEC. 1998.

BRETONES, P. S. \& MEGID NETO, J., Tendências de teses e dissertações sobre ensino de Astronomia no Brasil, In: REUNIÃO ANUAL DA SOCIEDADE ASTRONÔMICA BRASILEIRA, XXIX, 2003, Águas de São Pedro. Atas... Disponível em: $<$ http://www.fae.unicamp.br/cedoc/Tendencias_teses_astronomia.doc $>$, Acessado em Junho de 2009.

LANGHI, R. Um estudo exploratório para a inserção da Astronomia na formação de professores dos anos iniciais do ensino fundamental. 2004. 240 f. Dissertação (Mestrado em Educação para a Ciência). Faculdade de Ciências, UNESP, Bauru, 2004.

MARRONE JÚNIOR, J. Um perfil da pesquisa em ensino de Astronomia no Brasil a partir da análise de periódicos de ensino de Ciências. 2007. 253f. Dissertação (Mestrado em Ensino de Ciências e Educação Matemática) - Universidade Estadual de Londrina, Londrina, 2007.

OSTERMANN, F. \& MOREIRA, M. A., Uma revisão bibliográfica sobre a área de pesquisa "Física Moderna e Contemporânea no Ensino Médio", Investigações em Ensino de Ciências, v. 5, n. 1, p. 23-48, 2000. Disponível em <http://www.if.ufrgs.br/ienci>, acessado em Junho de 2009.

VASCONCELOS, C.; LOPES, B.; COSTA, N.; MARQUES, L. \& CARRASQUINHO, S., Estado da arte na resolução de problemas em Educação em Ciência. Revista Electrónica de Enseñanz̧a de las Ciencias, v. 6, n. 2, p. 235-245, 2007. Disponível em <http://www.saum.uvigo.es/reec>, acessado em Junho de 2009. 
